\title{
NUMERICAL COMPARISON OF ACTIVE ACOUSTIC AND STRUCTURAL NOISE CONTROL IN A STIFFENED DOUBLE WALL CYLINDER
}

\author{
Ferdinand W. Grosveld * \\ Lockheed Martin Engineering and Sciences \\ NASA Langley Research Center \\ Mail Stop 463 \\ Hampton, VA 23681
}

\begin{abstract}
$\underline{\text { Abstract }}$
The active acoustic and structural noise control characteristics of a double wall cylinder with and without ring stiffeners were numerically evaluated. An exterior monopole was assumed to acoustically excite the outside of the double wall cylinder at an acoustic cavity resonance frequency. Structural modal vibration properties of the inner and outer shells were analyzed by post-processing the results from a finite element analysis. A boundary element approach was used to calculate the acoustic cavity response and the coupled structural-acoustic interaction. In the frequency region of interest, below $500 \mathrm{~Hz}$, all structural resonant modes were found to be acoustically slow and the nonresonant modal response to be dominant. Active sound transmission control was achieved by control forces applied to the inner or outer shell, or acoustic control monopoles placed just outside the inner or outer shell. A least mean square technique was used to minimize the interior sound pressures at the nodes of a data recovery mesh. Results showed that single acoustic control monopoles placed just outside the inner or outer shells resulted in better sound transmission control than six distributed point forces applied to either one of the shells. Adding stiffeners to the double wall structure constrained the modal vibrations of the shells, making the double wall stiffer with associated higher modal frequencies. Active noise control obtained for the stiffened double wall configurations was less than for the unstiffened cylinder. In all cases, the acoustic control monopoles controlled the sound transmission into the interior better than the structural control forces.
\end{abstract}

\section{$\underline{\text { Introduction }}$}

The development of active noise control technology has progressed to the commercialization of systems using interior distributions of loudspeakers to achieve overall

\footnotetext{
*Supervisor, Aeroacoustics and Structural Acoustics Associate Fellow
}

Copyright $\odot 1996$ by F. W. Grosveld. Published by the American Institute of Aeronautics and Astronautics, Inc. with permission. noise reduction inside an aircraft cabin. ${ }^{1}$ Such systems generally require many acoustic control sources to obtain global cancellation since the local acoustic control field has inherently different modal properties than the primary field, which is driven by the distributed structural response of the cabin. ${ }^{2}$

Alternative methods have been studied for actively reducing aircraft interior noise at low frequencies. Appreciable sound attenuation was achieved across double wall structures by placing acoustic control sources in the space between the walls (loudspeakers between the fuselage and trim panel, for example). ${ }^{3}$ In other work, active structural acoustic control was investigated where force inputs were applied directly to the primary aircraft structure, thus eliminating the need to place acoustic control sources in the cabin. Such control forces might be produced by piezoelectric patches bonded to the structure. This approach has the potential to produce a global interior noise reduction with a limited number of control actuators. ${ }^{4,5}$ Additional reductions in the number of control actuators may be achieved by grouping the actuators to reduce control spillover into the structure. ${ }^{6,7}$ Amplitude and phase of the control forces can be optimized to maximize the noise reduction. ${ }^{8}$

In recent studies, the active structural acoustic control characteristics of a double wall cylinder with and without ring stiffeners were evaluated numerically using finite element/boundary element techniques. ${ }^{8,9,10}$ An exterior monopole was assumed to acoustically excite the outside of the double wall cylinder at an acoustic cavity resonance frequency. Structural modal vibration properties of the inner and outer shells were analyzed by post-processing the results from a finite element analysis. A boundary element approach was used to calculate the acoustic cavity response and the coupled structural-acoustic interaction. It was found that ring stiffeners couple the modal vibrations of the inner and 
outer shells, constrain their motions and shift their modal frequencies. ${ }^{10}$ For low-order modes the location of the ring stiffeners and the mode shapes of the double wall cylinder determined in-phase or out-of-phase motion of the individual shells. Out-of-phase shell motion made the double wall cylinder considerably stiffer, with associated higher modal frequencies. Inphase motion of the shells caused a less pronounced increase in the modal frequency or even lowered the frequencies of the shell dominant modal resonances. The extent of the shift of the modal frequencies determined how much sound was transmitted into the cavity and how much the interior sound pressure field could be reduced by actively controlling the vibration of the inner shell. This control was achieved by six optimized complex control forces, applied normal to the surface of the inner cylinder. This study ${ }^{10}$ is extended here by numerically investigating the active control of the sound transmission into the cylinder cavity by acoustic control monopoles between the cylinder shells at the same azimuth location as the previously used structural control forces. The active control of sound transmission is investigated for different structural force or acoustic monopole control arrangements in the unstiffened and stiffened double wall cylinder configurations.

\section{$\underline{\text { Structural and Acoustic Models }}$}

Two concentric cylindrical shells of length $\mathrm{L}=3.66 \mathrm{~m}$ are clamped at both ends by rigid end caps. The aluminum outer shell has a radius of $0.838 \mathrm{~m}$ and a thickness of $1.7 \mathrm{~mm}$. The composite inner shell has a radius of $0.780 \mathrm{~m}$, is $7.5 \mathrm{~mm}$ thick and has a Young's modulus of $16.9 * 10^{9} \mathrm{~Pa}$.

A cylindrical coordinate system $(\mathrm{x}, \mathrm{r}, \boldsymbol{\theta})$ was chosen with the origin in the center of one end cap and the $\mathrm{x}$ direction along the center line of the concentric cylinders. Considering the symmetry of the problem, only one quarter of the double cylinder needs to be modeled. Clamped boundary conditions were assumed at the end cap locations. The structural finite element model is depicted in Figure 1. The mesh representing each quarter shell consists of 20 QUAD4 elements in the longitudinal direction and 40 QUAD4 elements in the circumferential direction. Optional ring stiffeners with clamped edge conditions connect the two shells at L/6 and/or L/3 from one end cap. They consist of QUAD4 elements with aluminum material properties.

The primary interior acoustic field was generated by a monopole source of unit strength located $0.168 \mathrm{~m}$ from the outer shell at $\mathrm{x} / \mathrm{L}=0.5$ and $\theta=0$ degrees (Figure 1 , not to scale). Previously, six normal point control forces were applied to the inner shell at $\theta=0, \pm 31.5$, \pm 90 and 180 degrees in the center cross section to enable efficient control of the dominant interior acoustic responses at the frequency studied. ${ }^{10}$ For the current investigation, six acoustic control monopoles were placed at the same azimuth angles between the inner and outer shells at $0.809 \mathrm{~m}$ radius in the center cross section to study their effectiveness in controlling the sound transmission into the cylinder cavity. Single and multiple control forces applied to the inner or outer cylinder shell were evaluated and compared to acoustic control monopoles between the cylinder shells and at a radius of $0.867 \mathrm{~m}$, just $0.029 \mathrm{~m}$ outside the outer shell surface. Several other configurations were studied to compare active acoustic and structural control of the sound transmission into the double cylinder.

Sound pressures were numerically calculated at recovery grid points of horizontal, vertical and crosssectional meshes in a quarter model of the acoustic cavity. The horizontal and vertical acoustic meshes contain grids of 36 by 14 elements and 14 by 36 elements, respectively. Each cross section at $\mathrm{x} / \mathrm{L}=0.1$, $0.27,0.5,0.73$ and 0.9 has 640 grid points distributed over eight evenly spaced concentric circles. Full model results are presented for vertical and horizontal meshes and cross sectional meshes at $\mathrm{x} / \mathrm{L}=0.1, \mathrm{x} / \mathrm{L}=0.5$ and $\mathrm{x} / \mathrm{L}=0.73$ (Figure 1).

\section{Computational Procedures}

A normal mode analysis up to $500 \mathrm{~Hz}$ was performed in MSC/NASTRAN $^{\mathrm{TM}}$ to obtain the structural modal parameters of the unstiffened and stiffened double wall cylinder configurations. The modal data was analyzed in the post-processor PATRAN ${ }^{\mathrm{TM}}$ to obtain the mode shapes, longitudinal and circumferential mode numbers and phase information of the shell vibrations. An indirect boundary element method with coupled fluid/structural interaction was used in the commercial software package SYSNOISE ${ }^{\mathrm{TM}}$ to numerically calculate the acoustic pressures at the nodal points of the interior recovery mesh. Viscous damping $1 \%$ of critical was applied to the structural modes of the inner and outer cylinders. The primary field of interior sound pressures due to excitation by the exterior monopole source of unit strength was determined first, after which the control field for the unit control force at $\theta=0$ degrees was calculated. The interior acoustic pressure fields for the other control forces were obtained by rotating the response field to match the azimuth angles at which the control forces were applied. The same 
procedure was exercised to determine the interior acoustic pressure fields due to each of the monopole control sources.

A least mean square (LMS) algorithm ${ }^{11}$ was used to obtain the real and imaginary parts of the complex control force or acoustic control monopole that minimized the acoustic pressure inside the cylinder. The pressure for the primary and control fields was evaluated at eighty circumference recovery grid points $(\mathrm{r}=0.7 \mathrm{~m})$ in the three acoustic mesh cross-sections at $\mathrm{x} / \mathrm{L}=0.1,0.5$ and 0.73 . The control parameters were obtained by minimizing the sum of the squared pressures at these 240 pressure response points. The cost function ${ }^{9}$ approximates the total energy in the acoustic field. The controlled interior acoustic field is a linear superposition of the primary and control fields. The control monopoles do not interfere with each other or with the source monopole as only their responses at the nodal points of the interior recovery field are used in the superposition routine. The cost function reduction represents the decrease in acoustic energy when exercising optimized control of the primary acoustic field and is used in this study to gauge the effectiveness of the active sound transmission control into the cylinder.

\section{$\underline{\text { Unstiffened Double Wall Cylinder }}$}

\section{$\underline{\text { Acoustic Modal Frequencies }}$}

Acoustic modes were calculated for a hard wall interior cavity of the inner cylinder shell. A zero radial mode was assumed. The circumferential and longitudinal modes were determined from the post-processed acoustic modal data and are shown in Figure 2 as a function of the frequency. Mode numbers $\mathrm{n}$ denote the circumferential cosine order and mode number $\mathrm{m}$ indicates the number of nodes in the axial direction. Only cross-sectional $\cos (n \theta)$ resonances for the even axial modes are plotted. The odd axial modes will not be excited due to the symmetry of the problem.

\section{$\underline{\text { Structural Modal Frequencies }}$}

The inner and outer shells of the double wall cylinder are clamped at the rigid end caps and, without the ring stiffeners, are not structurally connected. Their invacuo modes of vibration were numerically calculated ${ }^{10}$ in MSC/NASTRAN ${ }^{\mathrm{TM}}$ and are summarized in Figure 3. Mode numbers are shown for modal frequencies below $400 \mathrm{~Hz}$. Mode number $\mathrm{n}$ refers to half the number of nodes along the circumference and $\mathrm{k}$ denotes the number of antinodes in the axial direction. Even axial modes will not be excited due to symmetry considerations.

\section{$\underline{\text { Double Cylinder Excitation }}$}

Monopole Source - The interior acoustic response was evaluated for a monopole oscillating at the $213.8 \mathrm{~Hz}$ resonance frequency of the $(2,0)$ acoustic cavity mode (Figure 2). The mechanism of the sound transmission through the cylinder walls depends on the type of excitation and its frequency in relation to the cutoff, ring and coincidence frequencies of the cylinder. ${ }^{12}$ Below the acoustic cutoff frequency only plane wave sound may be propagated inside the cylinder, since acoustic cross modes will decay exponentially with distance down the longitudinal axis (evanescent waves). The cutoff frequency $f_{c o}$ is given by the equation

$$
\mathrm{f}_{\mathrm{co}}=1.84 \mathrm{c}_{0} /(2 \pi \mathrm{r})
$$

where $\mathrm{r}$ is the shell radius and $\mathrm{c}_{0}$ is the speed of sound in $\operatorname{air}(340 \mathrm{~m} / \mathrm{s})$. Acoustic coincidence occurs when the in-vacuo bending wave speed equals the acoustic wave speed. The critical frequency is the lowest frequency at which coincidence occurs

$$
\mathrm{f}_{\mathrm{c}}=\mathrm{c}_{0}{ }^{2} /\left(2 \pi \kappa \mathrm{c}_{1}\right)
$$

where $\kappa=h / \sqrt{ } 12$ is the radius of gyration, $h$ is the thickness, $c_{l}=\sqrt{ }\left[E /\left\{\rho\left(1-\mu^{2}\right)\right\}\right]$ is the longitudinal wave speed and $\mu$ is Poisson's ratio. Above the coincidence frequency the resonant modes are acoustically fast and radiate sound over the total surface area with a radiation efficiency close to unity. Acoustically slow resonant modes, below the coincidence frequency, couple to the acoustic interior only by inefficient wall modes and the radiation efficiency gets smaller with decreasing frequency. In this region non-resonant mass-controlled shell motion is the dominant mechanism for the radiation of sound into the interior. At the ring frequency $f_{R}$ the longitudinal wavelength equals the circumference of the cylinder

$$
\mathrm{f}_{\mathrm{R}}=\mathrm{c}_{\mathrm{L}} /(2 \pi \mathrm{r})
$$

where $c_{L}=\sqrt{ }(E / \rho)$ is the bar wave speed, $E$ is the Young's modulus and $\rho$ is the density. Below the ring frequency the motion around the circumference is constrained by the extensional stiffness around the circumference (curvature effect). At frequencies well above the ring frequency the motion of the cylinder shell is much like the flexural behavior of a flat plate. The cutoff, ring and critical coincidence frequencies were calculated from the geometrical and material shell properties and are listed in Table 1 . The $(2,0)$ acoustic 
modal frequency $(213.8 \mathrm{~Hz})$ is above the cutoff frequency, below the ring frequency and well below the critical frequency. Acoustically fast modes only exist when their structural wavenumbers $k_{\mathrm{nk}}$ are smaller than the acoustic trace wavenumber at the resonance frequencies of those modes

$$
k^{2} \geq k_{\mathrm{nk}}^{2}=k_{\mathrm{n}}^{2}+k_{\mathrm{k}}^{2}
$$

where $k, k_{\mathrm{n}}=\mathrm{n} / 2 \mathrm{r}$ and $k_{\mathrm{k}}=\mathrm{k} \pi \mathrm{L}$ are the acoustic, shell axial and shell circumferential wavenumbers, respectively. In the frequency region of interest, below $500 \mathrm{~Hz}$, all structural resonant modes are acoustically slow and the non-resonant modal response is dominant.

The normalized pressure distribution on the interior field recovery mesh resulting from the excitation by the monopole at $213.8 \mathrm{~Hz}$ is depicted in Figure 4. The pressure distribution is normalized with respect to the excitation by an acoustic monopole of unit strength. The interior response is mostly through the $(2,0)$ acoustic mode, as evidenced by the pressure distribution in the cross-sectional (center) and horizontal meshes (Figures 5a and 5b). For this figure and subsequent figures the source or controller is always located in the center below the cross-sectional or horizontal mesh. The $(2,0)$ acoustic mode couples well with the offresonance forced response of the $(2,1)$ outer shell mode, $\mathrm{O}(2,1)$, which resonates at $154.9 \mathrm{~Hz}$ (Figure 3). A $43.5 \%$ participation factor (PF), which is the ratio of the modal displacement amplitude relative to the total structural response, was calculated for the outer $\mathrm{O}(2,1)$ shell mode (Table 2). The $\mathrm{O}(7,5)$ outer shell mode participates for $5.9 \%$ in the total structural response while the $\mathrm{I}(5,3)$ inner shell mode contributes for $5 \%$. Only the modes with a participation factor of at least $5 \%$ are listed in Table 2. The inner and outer shells are only coupled by the acoustic medium in the annular space between the shells. For most angles of sound incidence the dimension of this space, along the path of the incoming sound, is much smaller than the $1.59 \mathrm{~m}$ acoustic wavelength at $213.8 \mathrm{~Hz}$ and the inner and outer shells are forced to move together. The sound transmission into the cylinder increases with increasing angle of sound incidence from the normal. Axial acoustic modes of the fourth order are forced on the source side of the double cylinder where they match the sound pattern over the exterior of the sidewall coming directly from the acoustic source monopole (Figure $5 \mathrm{~b}$ ). The $(0,4),(1,4)$ and $(2,4)$ modes are resonant at 187.4 $\mathrm{Hz}, 227.5 \mathrm{~Hz}$ and $284.3 \mathrm{~Hz}$, respectively, and are susceptible to be excited (Figure 2). The interior sound pressure in the vertical recovery mesh is, of course, symmetrical about the longitudinal axis and shows a mixture of lower order axial acoustic modes (Figure 6).
Control Forces and Control Monopoles - The sound transmitted into the double cylinder is actively controlled by either one or more point forces or acoustic monopoles. The mechanism of the sound transmission is dependent on the excitation frequency as discussed in the previous section but also on the nature of the excitation. A structural point force or an airborne sound field excite a structure differently. ${ }^{12}$ The sound power transmitted by a fluctuating structural force consists of the contributions of a near-field component and a reverberant component. The near-field part radiates acoustic power from the location where the point force is applied since the shell velocity in the vicinity of the driving point has no counterpart for volume velocity cancellation. The reverberant field radiates sound through corner, edge and strip modes. The total radiation is the sum of the forced radiation in the nearfield plus the free modal response from the reverberant field. Mainly higher order modes resonant around the driving force frequency are excited. For this investigation the inner shell was excited by a point force in the center cross section (configuration F1) and the horizontal plane $(\theta=0)$. Modes having a participation factor greater than 5\% are listed in Table 2. Mostly the higher-order, third axial inner shell modes around the excitation frequency are forced to participate. The structural modes couple with the acoustic modes in the interior. The normalized acoustic pressure response for the center cross-sectional and horizontal meshes are depicted in Figure 7. The pressure distribution is normalized with respect to the excitation by a unit force input. In addition to the $(2,0)$ acoustic mode the crosssectional mesh shows contributions of the higher-order circumferential structural modes. Applying the point force to the outer shell in the center cross section (configuration F2) and the horizontal plane induces higher-order outer shell modes around the $213.8 \mathrm{~Hz}$ excitation frequency. These modes and their participation factors are listed in Table 2. Mainly the $(2,0)$ acoustic mode is excited in the F2 configuration, but is not shown here.

Excitation by an acoustic monopole located in the center cross-section between the inner and outer shells (configuration M1) at $\theta=0$ forces the participation of the same $\mathrm{O}(2,1)$ and $\mathrm{I}(5,3)$ modes as for excitation by the external source monopole. However, instead of creating an external pressure distribution over the outer shell, it forces sound directly through the inner shell into the acoustic interior. The acoustic response from the $(2,0)$ mode is almost symmetric (Figure 8 ), as its wavelength $(1.59 \mathrm{~m})$ almost matches the diagonal interior dimension of the acoustic cavity (1.56). This was not the case for excitation by the exterior source 
monopole (Figure 5). For configuration M2, where an acoustic monopole is located in the center cross-section just outside $(\mathrm{r}=0.867 \mathrm{~m})$ the outer shell at $\theta=0$ degrees, the $\mathrm{O}(2,1)$ is again the highest contributing mode $(\mathrm{PF}=21.2 \%)$. The major structural modes participating $(\mathrm{PF}>5 \%)$ are listed in Table 2. The interior acoustic response, which is not plotted here, is very similar to the pressure distribution induced by the exterior source monopole but with higher interior pressure amplitudes.

\section{$\underline{\text { Cost Function Reductions }}$}

Cost function reductions were calculated for several configurations where the sound transmission from the external source monopole was actively reduced by point forces on the inner or outer shell (F1 and F2) or acoustic monopoles just outside the inner or outer shells (M1 and M2). Table 3 lists the cost function reductions for the four configurations. The controllers are located in the same horizontal plane as the external source monopole. The transmission loss characteristics of both the inner and outer shells modify the sound generated by the exterior source monopole before it reaches the interior. Sound generated by the point control force on the outer shell is altered by the outer shell structural vibration and the inner shell transmission loss characteristics when it arrives in the cylinder cavity. However, a point force on the inner shell directly radiates sound into the interior and is not affected by the structural vibration properties of the outer shell. The cost function reduction by the point control force on the outer shell was $6.70 \mathrm{~dB}$ compared to the $2.24 \mathrm{~dB}$ for the control exercised by the point force on the inner shell.

The cost function reduction of $2.24 \mathrm{~dB}$ for one point force controller on the inner shell was increased to 6.39 $\mathrm{dB}$ (Table 3 ) by applying six point force controllers at angles $\theta=0, \pm 31.5, \pm 90$ and 180 degrees on the inner shell. The lack of contributions in the interior acoustic response from the outer shell vibration was thus compensated for by using six point forces instead of one. The normalized interior pressure distribution when excited by the source monopole but controlled by the six point forces on the inner shell is depicted in Figure 9. The sound transmission control by six point forces at the same azimuth locations on the outer shell resulted in a cost function reduction of $6.92 \mathrm{~dB}$ (Table 3). This is only $0.22 \mathrm{~dB}$ more than the cost function reduction achieved by a single point control force. The interior acoustic pressure distribution is thus not much improved by using the six point force controllers instead of one to reduce the sound transmission into the interior.
The cost function reduction achieved by the monopole located just outside the outer shell was significantly higher than the cost function reduction obtained by the monopole between the two cylinder shells (29.3 dB versus $6.99 \mathrm{~dB}$ in Table 3). Sound from the exterior source and control monopoles radiated into the interior are modified by the transmission loss characteristics of both the inner and outer shells when radiated into the interior. Sound from the control monopole between the two shells reaching the cylinder cavity will be affected by mainly the inner shell. The interior sound pressure fields due to the exterior source and control monopoles will be more alike and result in better sound transmission control. A single acoustic control monopole just outside the inner or outer shells resulted in a higher cost function reduction than obtained through the structural noise control by six point forces (Table 3).

The six acoustic control monopoles distributed between the inner and outer shells at the same azimuth angles where previously the control forces were applied achieved a $3.6 \mathrm{~dB}$ higher cost function reduction than a single control monopole. Placing six distributed acoustic control monopoles along the outside perimeter of the outer shell resulted in a $4.7 \mathrm{~dB}$ higher cost function reduction than was obtained for a single control monopole. Applying point control forces to the outer shell or having exterior acoustic controllers is often impractical, impossible or not permitted, especially in aircraft. These configurations are not further reported on in this paper. Active control by a single point force or acoustic monopole was investigated for each of the previously used azimuth angles. Figure 5a shows the second circumferential acoustic mode due to excitation by the external monopole source. The cross-sectional pressure distribution shows local maxima at $\theta=0, \theta= \pm 90$ and $\theta=180$ degrees. Applying active controllers at each of these radial locations provides approximately the same cost function reductions but the acoustic monopole controllers perform more than $4 \mathrm{~dB}$ better than the point control forces (Table 4). The cost function reductions are significantly lower when these controllers are applied at \pm 31.5 degrees, where sound pressure amplitudes are at a local minimum (Figure 5a). Cost function reductions for two controllers at $\theta=0$ and $\theta=180$ degrees and four controllers at $\theta=0, \pm 90$ and 180 degrees were also computed and are listed in Table 4. The acoustic control monopoles outperform the structural control point forces in actively controlling the sound transmission into the interior. 


\section{$\underline{\text { Stiffened Double Wall Cylinder Configurations }}$}

\section{$\underline{\text { Structural Modal Frequencies }}$}

When the inner and outer shells are joined by ring stiffeners the previously independent shell modes become coupled. Some of the modal vibration response dominant in one shell will be present in the other shell and vice versa. Coupled modes with a dominant modal response of the inner or the outer cylinder are referred to as the inner or outer shell dominant modes, respectively. Modal frequencies and mode numbers of the inner and outer shell dominant modes for the configurations featuring ring stiffeners at distances $\mathrm{L} / 6$ or/and L/3 from the endcaps (configuration RX, XS, and RS, respectively) are presented in Figures 10a, 10b and 10c. Adding stiffeners to the double wall structure generally constrains the modal vibrations of the shells, making the double wall stiffer with associated higher modal frequencies. It was found in Reference 10 that, for the lower-order modes (up to the fifth circumferential mode), this shift in frequency is considerably higher when the inner and outer shells move out of phase than when they move together. The phase of the shell motions was found to be the same for all dominant lower-order inner shell modes and opposite in phase for all dominant lower-order outer shell modes. ${ }^{10}$ Configuration RX, having stiffeners at L/6 from the end caps, was found to have in-phase motion of the outer and inner shells causing only a modest shift in modal frequency for the lower-order modes. The $\mathrm{O}(2,1)$ modal frequency shifted from 154.9 $\mathrm{Hz}$ for the unstiffened double cylinder (Figure 3) to $172.8 \mathrm{~Hz}$ for the RX configuration (Figure 10a). The double cylinder configurations with stiffeners at $\mathrm{L} / 3$ from the end caps have out-of-phase shell motion of the lower-order $\mathrm{O}(2,1)$ mode, causing a significant shift in frequency to $481.9 \mathrm{~Hz}$ for the XS configuration and to $484.3 \mathrm{~Hz}$ for configuration RS. These shifts in modal frequencies are important to determine the structural modal participation in the sound transmission process. The lowest modal frequencies in Figures 10a, 10b and $10 \mathrm{c}$ are for in-phase shell motion of the lower-order inner or outer shell modes.

\section{Excitation of the RX Stiffened Double Cylinder}

Monopole Source - Modes participating for more than $5 \%$ in the shell vibration of the stiffened double cylinder are listed in Table 5. The RX outer shell dominant $\mathrm{O}(2,1)$ mode has a $\mathrm{PF}$ of $22.2 \%$ at $172.8 \mathrm{~Hz}$ when excited by the exterior source monopole, which is about half the $43.5 \%$ participation factor for the unstiffened cylinder at $154.9 \mathrm{~Hz}$ (Table 2). The $\mathrm{O}(10,5)$ and $\mathrm{O}(6,3)$ outer shell dominant modes also contribute substantially to the total double wall vibration. The $\mathrm{O}(2,1)$ mode couples to the acoustic $(2,0)$ mode which can be seen in Figure 11, where the interior pressure distribution at the nodes of the field recovery mesh is depicted. The second circumferential acoustic mode is present in the cross section closest to one of the endcaps. This mode is dominant only in the part of the cavity between the endcaps and the stiffeners. Between the stiffeners, the shell vibrations seem to couple to the acoustic $(1,4)$ mode which is resonant at $227.5 \mathrm{~Hz}$. The center cross section of the interior pressure distribution in Figure 12a shows a first-order circumferential mode. The horizontal plane in that figure shows that the distance between the two prominent nodal lines is close to $\mathrm{L} / 4$, suggesting a fourth-order axial acoustic mode. The pressure levels are normalized to the monopole excitation.

Control Forces and Control Monopoles - Mostly higherorder third axial inner shell modes were excited when a control point force was applied to the inner shell of the unstiffened cylinder (configuration F1 in Table 2). The addition of ring stiffeners, however, shifted the third axial inner shell dominant modes to well above the excitation frequency of $213.8 \mathrm{~Hz}$ (Figure 10a). The RX stiffened double cylinder is now responding through higher-order first axial modes when excited by the point force (Table 5). The normalized interior pressure field response is shown in Figure $12 \mathrm{~b}$ for the center cross section and the horizontal plane. The control point force excitation is applied to the bottom center of both the cross section and the horizontal plane. The first axial mode is clearly distinguishable, but not the higherorder circumferential modes. The sound transmission mainly takes place between the two ring stiffeners at the side of the double cylinder where the point force is located. Low sound pressure levels are apparent in the regions close to the endcaps.

The acoustic control monopole between the inner and outer shells strongly excites the acoustic $(2,0)$ mode (Figure 12c) through the $\mathrm{O}(2,1)$ and $\mathrm{I}(2,1)$ structural modes (Table 5). The stiffeners in the RX configuration restrict the structural vibration of the coupled inner and outer shells, resulting in lower maximum normalized pressures $\left(\mathrm{p}_{\max }=16.0\right.$ in Figure $12 \mathrm{c}$ compared to $p_{\max }=40.0$ in Figure 8).

\section{Excitation of the XS and RS Stiffened Double Cylinders}

The interior acoustic response to the excitation of XS and RS stiffened double cylinders is similar, differing mostly in the details of the pressure distribution and the response/excitation ratios. Only the acoustic excitation responses of the RS configuration are presented here. 
Monopole Source Excitation - Both the XS and RS stiffened double cylinders showed the forced interior acoustic response on the side where the cylinder was directly exposed to the acoustic source monopole. This is depicted in Figure 13a for the RS configuration. The source monopole was located in the center below the cross-sectional and horizontal illustrations of the double cylinder. The first four modes participating in the shell vibrations of the RS cylinder are first-order axial inner and outer shell dominant modes (Table 5). This is consistent with the even pressure distribution in the horizontal plane opposite the side of the source excitation. The acoustic $(2,0)$ mode was excited but showed only in the region close to the endcaps, similar to the response for the RX cylinder in Figure 11.

Control Forces and Control Monopoles - The structural response to the control point force on the inner shell is mostly through the higher-order first axial inner shell dominant modes (Table 5). The acoustic response to the higher circumferential structural modes is visible in the cross-sectional pressure distribution of Figure 13b. The acoustic response through the $(2,0)$ mode is also apparent from the cross-sectional and axial sound pressure distributions in Figure 13b. Sound pressure levels in the regions close to the endcaps are low.

The interior pressure distribution due to the excitation by the control acoustic monopole between the inner and outer shells is depicted in Figure 13c. The maximum normalized pressure ratio is considerably down $\left(\mathrm{p}_{\max }=3.2\right)$ compared to the excitation of the unstiffened $\left(p_{\max }=40.0\right)$ and $\mathrm{RX}$ stiffened $\left(\mathrm{p}_{\max }=16.0\right)$ double wall cylinders. Unattenuated excitation of the cylinder only takes place between the innermost ring stiffeners which are only L/3 apart. Some of the same higher-order first axial modes as excited by the control point force are participating. However the acoustic excitation also induces outer shell dominant modes (Table 5). The higher-order structural circumferential modes show in the center cross-sectional acoustic response in Figure $13 \mathrm{c}$.

\section{Cost Function Reductions}

In Table 6 the cost function reductions of the stiffened (RX, XS and RS) and the unstiffened (XX) double wall cylinder configurations are compared for excitation by one (F1) or six (F6) point control forces or one (M1) or six (M6) acoustic control monopoles. For the unstiffened double wall cylinder configuration the source monopole and the control force both excited the $\mathrm{I}(5,3)$ mode (Table 2) and their cross-sectional and axial pressure distributions (Figures 5 and 7) were similar over a small portion of the interior acoustic space, resulting in a cost function reduction of $2.24 \mathrm{~dB}$. The source monopole and the point control force exciting the RX stiffened cylinder did not have a structural mode in common with a participation factor higher than 5\% (Table 5). The interior acoustic pressure distributions in Figures $12 \mathrm{a}$ and $12 \mathrm{~b}$ do not correspond very well and the cost function reduction obtained by the single point control force was only 0.16 $\mathrm{dB}$ (Table 6). The acoustic source monopole and the point control force in the XS and RS configurations did not excite the same structural mode either, but both invoked some first-order axial inner shell dominant modes (Table 5). Their acoustic interior pressure distributions (Figures 13a and 13b) seemed to correspond somewhat better on the excitation side of the cylinder interior, resulting in a slightly higher cost function reduction (Table 6).

Both the source and the control monopoles excited the $\mathrm{O}(2,1)$ and the $\mathrm{I}(5,3)$ structural modes of the unstiffened double cylinder (Table 1). The high participation factors and the well matching acoustic interior pressure distributions (Figures 5 and 8) resulted in a $6.99 \mathrm{~dB}$ cost function reduction. Source and control excitations of the RX stiffened configuration have the $\mathrm{O}(2,1)$ and $\mathrm{O}(6,3)$ structural modes in common (Table 5). The $\mathrm{O}(4,3)$ is the commonly excited mode in the XS configuration while the $\mathrm{O}(4,1)$ structural mode is excited by both the source monopole and control monopole in the RS configuration. However, their participation factors are considerably less than the participation factors of the common structural modes for the unstiffened cylinder (Tables 2 and 5). The RX and RS acoustic responses to the source and control monopole excitation agree more (Figures 12a and 12c, $13 \mathrm{a}$ and $13 \mathrm{c}$ ) than the interior pressure distributions due to the source monopole and the point control forces (Figures 12a and 12b, 13a and 13b). The control monopole excitation is restricted to a smaller area of the double cylinder due to the ring stiffeners and does not match the interior acoustic response field as well as for the unstiffened double cylinder case. Similar observations explain that the cost function reductions for the XS configuration are lower than for the unstiffened double cylinder case. For all configurations (Table 6) higher cost function reductions were obtained for the six control monopoles than for the six point control forces. For the XS and RS stiffened double wall cylinders, the superposition of the acoustic response fields from the six point control forces is less effective in controlling the sound transmission than the superposition of the interior pressure distributions from the six acoustic control monopoles (Table 6). 


\section{$\underline{\text { Conclusions }}$}

The double wall cylinder was excited by a source monopole at the $213.8 \mathrm{~Hz}$ modal frequency of the $(2,0)$ interior acoustic mode. This excitation frequency was calculated to occur above the interior cavity cutoff frequency, below the ring frequency and well below the acoustic critical frequency. In the frequency region of interest, below $500 \mathrm{~Hz}$, all structural resonant modes were found to be acoustically slow and the nonresonant modal response was dominant. The acoustic pressures at 240 evenly spaced azimuth locations were numerically calculated in three cross sections of the acoustic recovery mesh. Active sound transmission control was achieved by applying control forces to the inner or outer shell or by placing acoustic control monopoles just outside the inner or outer shell. A least mean square technique was used to minimize the interior sound. The acoustic monopoles force the structure over an area and excite modes that couple best with the acoustic interior. The point control force excites the structure at a point inducing mostly higherorder structural modes around the excitation frequency. The interior pressure distribution due to the control monopole matched the interior acoustic response forced by the source monopole better than the point control force. Results showed that single acoustic control monopoles just outside the inner or outer shells resulted in higher cost function reductions than control by six point forces. The excitation of the acoustic source monopole induced a second-order circumferential modal pattern with sound pressure amplitudes having a local maximum at $\theta=0, \theta= \pm 90$ and $\theta=180$ degrees. An acoustic control monopole placed at one of these azimuthal locations produced a $4 \mathrm{~dB}$ higher cost function reduction than obtained by using a point control force. The cost function reduction is significantly lower when these controllers are applied at \pm 31.5 degrees, where sound pressure amplitudes are close to a local minimum. Adding stiffeners to the double wall structure generally constrains the modal vibrations of the shells, making the double wall stiffer with associated higher modal frequencies. Some of the modes will then no longer participate in the sound transmission process and affect the cost function reductions. Lower cost function reductions were obtained for the stiffened than for the unstiffened double wall configurations. The acoustic control monopoles outperformed the structural control forces for all cases in actively controlling the sound transmission into the interior.

\section{$\underline{\text { Acknowledgment }}$}

This study was supported by NASA Langley Research Center under Contract NAS1-19000, Dr. Kevin P. Shepherd, Task Manager.

\section{References}

1. J.T. McKenna, "Airline Outlook, Silencing Saab 340s," Aviation Week and Space Technology, February 1, 1993, p. 17.

2. S.J. Elliott, P.A. Nelson, I. Stothers and C. Boucher, "In-flight Experiments on the Active Control of Propeller-Induced Cabin Noise," J. Sound and Vibration, (140), 1990, pp. 219-238.

3. F.W. Grosveld and K.P. Shepherd, "Active Sound Attenuation Across a Double Wall Structure," Journal of Aircraft, vol. 31, no. 1, January-February 1994, pp. 223-228.

4. C.R. Fuller, S. Snyder, C. Hansen and R.J. Silcox, "Active Control of Interior Noise in Model Aircraft Fuselages using Piezoceramic Actuators," AIAA Journal, vol. 30, no. 11, 1992, pp. 2613-2617.

5. R.J. Silcox, S. Lefebvre, V.L. Metcalf, T.B. Beyer and C.R. Fuller, "Evaluation of Piezoceramic Actuators for Control of Aircraft Interior Noise," AIAA Paper No. 92-02-091, Proceedings of DGLR/AIAA 14th Aeroacoustics Conference, May 11-14, 1992, Aachen, Germany, Vol. II, pp. 542-551.

6. H.C. Lester and S. Lefebvre, "Piezoelectric Actuators Models for Active Sound and Vibration Control of Cylinders," Proceedings of Recent Advances in Active Control of Sound and Vibration, Blacksburg, VA, April 15-17, 1991, pp. 3-26.

7. R.H. Cabell, H.C. Lester and R.J. Silcox, "The Optimization of Force Inputs for Active Structural Acoustic Control using a Neural Network," Proceedings of Inter-Noise 92, Toronto, Ontario, Canada, vol. I, July 20-22, 1992, pp. 401-404.

8. T. Coats, R.J. Silcox and H.C. Lester, "Numerical Study of Active Structural Acoustic Control in a Double-Walled Cylinder," Proceedings of Recent Advances in Active Control of Sound and Vibration, Blacksburg, VA, April 28-30, 1993.

9. F.W. Grosveld, T.J. Coats, H.C. Lester, and R.J. Silcox, "A Numerical Study of Active Structural Acoustic Control in a Stiffened, Double Wall Cylinder," Proceedings of NOISE-CON 94, National Conference on Noise Control Engineering, Ft. Lauderdale, FL, May 1-5, 1994, pp. 404-408.

10. F.W. Grosveld and H.C. Lester, "Numerical Study of Active Sound Transmission Control for Stiffened Double Wall Cylinders," presented at the 1995 International Symposium on Active Control of Sound and Vibration, ACTIVE 95, Newport Beach, California, July 06-08, 1995.

11. H.C. Lester and C.R. Fuller, "Active Control of Propeller Induced Noise Fields Inside a Flexible Cylinder," AIAA Journal, vol. 28, no 8, August 1990.

12. W.K. Blake, Mechanics of Flow-Induced Sound and Vibration, Volume II, Academic Press, Inc,. 1986, pp. 658-690. 
Table 1. Double wall cylinder material and acoustic properties

\begin{tabular}{ccccccccc}
\hline & $\mathrm{a}$ & $\mathrm{h}$ & $\rho$ & $\mathrm{E}$ & $\mu$ & $\mathrm{f}_{\mathrm{co}}$ & $\mathrm{f}_{\mathrm{R}}$ & $\mathrm{f}_{\mathrm{c}}$ \\
& {$[\mathrm{m}]$} & {$[\mathrm{m}]$} & {$\left[\mathrm{kg} / \mathrm{m}^{3}\right]$} & {$[\mathrm{Pa}]$} & {$[-]$} & {$[\mathrm{Hz}]$} & {$[\mathrm{Hz}]$} & {$[\mathrm{Hz}]$} \\
\hline & & & & & & & & \\
Outer Shell & 0.838 & 0.0017 & 2700 & $71 * 10^{9}$ & 0.33 & 119 & 974 & 6983 \\
Inner Shell & 0.780 & 0.0075 & 394.4 & $16.9 * 10^{9}$ & 0.25 & 128 & 1336 & 12718 \\
\hline
\end{tabular}

Table 2. Mode participation for different excitation configurations

\begin{tabular}{lllll}
\hline Excitation & Location & Participating & Participation \\
& $(\theta=0)$ & Mode & Factor (PF) & $\begin{array}{l}\text { Frency } \\
{[\mathrm{Hz}]}\end{array}$ \\
\hline Monopole Source & External & $\mathrm{O}(2,1)$ & 43.5 & 154.9 \\
& (Configuration Source) & $\mathrm{O}(7,5)$ & 5.9 & 229.0 \\
& & $\mathrm{I}(5,3)$ & 5.0 & 244.3 \\
Point Force & On the Inner Shell & $\mathrm{I}(5,3)$ & 21.1 & 244.3 \\
& (Configuration F1) & $\mathrm{I}(6,3)$ & 15.8 & 224.3 \\
& & $\mathrm{I}(7,3)$ & 14.7 & 240.6 \\
& & $\mathrm{O}(5,3)$ & 8.0 & 177.5 \\
Point Force & On the Outer Shell & $\mathrm{I}(8,1)$ & 7.0 & 249.8 \\
& (Configuration F2) & $\mathrm{O}(15,7)$ & 14.4 & 212.6 \\
& & $\mathrm{O}(17,5)$ & 9.3 & 219.8 \\
Acoustic Monopole & $\mathrm{O}(11,7)$ & 5.8 & 219.9 \\
& & $\mathrm{O}(7,5)$ & 5.6 & 229.0 \\
& Between Inner and Outer Shells & $\mathrm{O}(2,1)$ & 23.0 & 154.9 \\
Acoustic Monopole & (Configuration M1) & $\mathrm{I}(5,3)$ & 8.2 & 244.3 \\
& & $\mathrm{O}(11,7)$ & 5.0 & 219.9 \\
& Slightly Outside the Outer Shell & $\mathrm{O}(2,1)$ & 21.2 & 154.9 \\
& (Configuration M2) & $\mathrm{O}(11,7)$ & 10.6 & 219.9 \\
& & $\mathrm{O}(7,5)$ & 8.4 & 229.0 \\
\hline
\end{tabular}

Table 3. Cost function reductions [dB] for control configurations F1, F2, M1 and M2

\begin{tabular}{ccc}
\hline Configuration & One Controller & Six Controllers \\
\hline F1 & 2.24 & 6.39 \\
F2 & 6.70 & 6.92 \\
M1 & 6.99 & 10.6 \\
M2 & 29.3 & 34.0 \\
\hline
\end{tabular}

Table 4. Cost function reduction $[\mathrm{dB}]$ for control configurations $\mathrm{F} 1$ and $\mathrm{M} 1$ at several azimuthal locations

\begin{tabular}{lcccccc}
\hline $\begin{array}{l}\text { Radial Location } \\
\text { [degrees] }\end{array}$ & $\theta=0$ & $\theta=31.5$ & $\theta=90$ & $\theta=180$ & $\theta=0,180$ & $\theta=0, \pm 90,180$ \\
\hline Configuration F1 & 2.24 & 0.37 & 2.29 & 2.32 & 4.60 & 4.78 \\
Configuration M1 & 6.99 & 0.80 & 6.73 & 6.44 & 8.38 & 9.95 \\
\hline
\end{tabular}


Table 5. Mode participation in the stiffened double cylinder configurations

\begin{tabular}{|c|c|c|c|c|c|c|c|c|c|}
\hline \multicolumn{2}{|c|}{ Configuration } & \multicolumn{2}{|l|}{$\mathrm{RX}$} & \multicolumn{3}{|c|}{ XS } & \multicolumn{3}{|c|}{$\mathrm{RS}$} \\
\hline & Mode & $\begin{array}{c}\mathrm{PF} \\
{[\%]}\end{array}$ & $\begin{array}{l}\text { Freq } \\
{[\mathrm{Hz}]}\end{array}$ & Mode & $\begin{array}{c}\mathrm{PF} \\
{[\%]}\end{array}$ & $\begin{array}{l}\text { Freq } \\
{[\mathrm{Hz}]}\end{array}$ & Mode & $\begin{array}{c}\mathrm{PF} \\
{[\%]}\end{array}$ & $\begin{array}{l}\text { Freq } \\
{[\mathrm{Hz}]}\end{array}$ \\
\hline \multirow[t]{6}{*}{ Source } & $\mathrm{O}(2,1)$ & 22.2 & 172.8 & $\mathrm{I}(2,1)$ & 15.9 & 175.1 & $\mathrm{I}(4,1)$ & 11.6 & 166.4 \\
\hline & $\mathrm{O}(10,5)$ & 19.3 & 219.0 & $\mathrm{O}(4,3)$ & 10.4 & 268.6 & $\mathrm{I}(3,1)$ & 9.4 & 139.4 \\
\hline & $\mathrm{O}(6,3)$ & 6.8 & 237.8 & $\mathrm{I}(4,1)$ & 8.8 & 159.9 & $\mathrm{O}(4,1)$ & 8.1 & 234.3 \\
\hline & & & & $\mathrm{I}(4,3)$ & 5.9 & 314.6 & $\mathrm{O}(5,1)$ & 7.4 & 163.1 \\
\hline & & & & $\mathrm{I}(3,1)$ & 5.8 & 133.9 & $\mathrm{O}(14,5)$ & 5.4 & 220.1 \\
\hline & & & & $\mathrm{O}(5,1)$ & 5.0 & 158.7 & $\mathrm{O}(13,5)$ & 5.0 & 218.9 \\
\hline \multirow[t]{4}{*}{ F1 } & $\mathrm{I}(8,1)$ & 16.7 & 254.1 & $\mathrm{I}(7,1)$ & 51.7 & 233.0 & $\mathrm{I}(7,1)$ & 39.5 & 238.4 \\
\hline & $\mathrm{I}(7,1)$ & 14.8 & 196.3 & $\mathrm{I}(6,1)$ & 8.6 & 209.3 & $\mathrm{I}(6,1)$ & 16.4 & 213.3 \\
\hline & $\mathrm{I}(6,1)$ & 6.4 & 151.0 & $\mathrm{O}(7,1)$ & 5.0 & 202.0 & $\mathrm{I}(5,1)$ & 7.4 & 206.0 \\
\hline & & & & & & & $\mathrm{I}(8,1)$ & 5.9 & 282.8 \\
\hline \multirow[t]{5}{*}{ M1 } & $\mathrm{I}(2,1)$ & 10.3 & 263.6 & $\mathrm{I}(7,1)$ & 24.4 & 233.0 & $\mathrm{I}(7,1)$ & 16.4 & 238.4 \\
\hline & $\mathrm{O}(2,1)$ & 8.8 & 172.8 & $\mathrm{O}(4,1)$ & 7.7 & 225.0 & $\mathrm{O}(4,1)$ & 12.4 & 234.3 \\
\hline & $\mathrm{O}(6,3)$ & 8.5 & 237.8 & $\mathrm{I}(6,1)$ & 5.8 & 209.3 & $\mathrm{I}(6,1)$ & 9.7 & 213.3 \\
\hline & $\mathrm{I}(3,1)$ & 5.3 & 157.0 & $\mathrm{O}(4,3)$ & 5.7 & 268.6 & $\mathrm{I}(5,1)$ & 8.3 & 206.0 \\
\hline & $\mathrm{O}(17,3)$ & 5.3 & 215.8 & $\mathrm{O}(3,1)$ & 5.2 & 319.2 & $\mathrm{O}(3,1)$ & 7.7 & 324.1 \\
\hline
\end{tabular}

Table 6. Cost function reductions $[\mathrm{dB}]$ of the unstiffened and stiffened double wall cylinders

\begin{tabular}{lllll}
\hline Configuration & XX & RX & XS & RS \\
\hline F1 (One Control) & 2.24 & 0.16 & 0.93 & 1.74 \\
M1 (One Control) & 6.99 & 3.38 & 1.15 & 2.91 \\
F1 (Six Controls) & 6.39 & 4.86 & 2.92 & 2.87 \\
M1 (Six Controls) & 10.6 & 5.17 & 6.25 & 8.71 \\
\hline
\end{tabular}

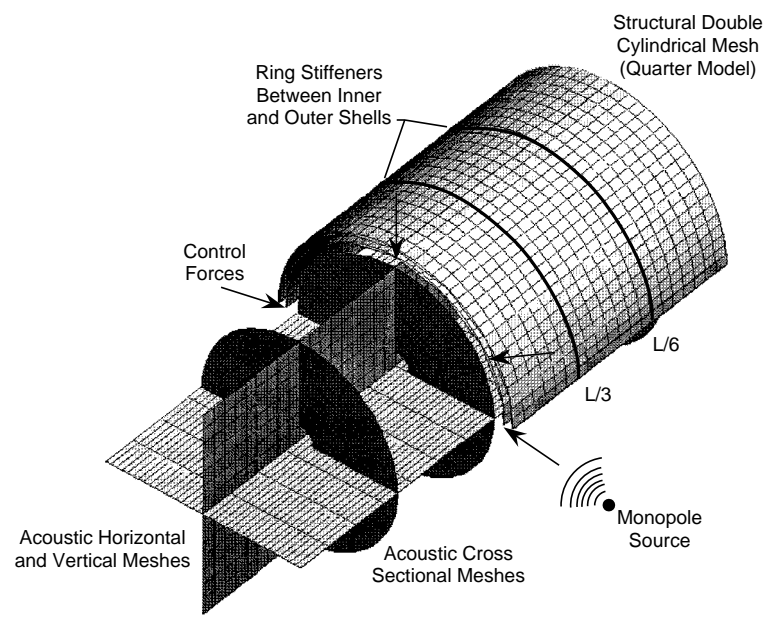

Figure 1. Structural and acoustic meshes of the double wall cylinder

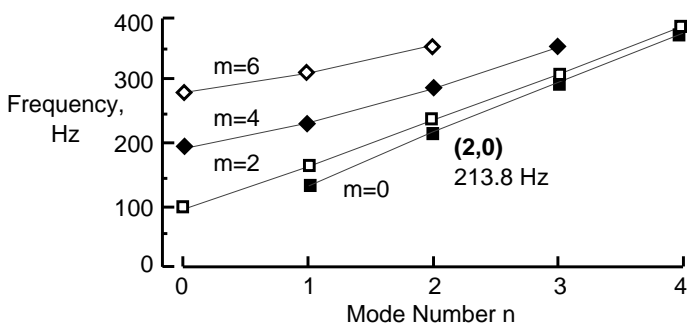

Figure 2. Acoustic circumferential (n) and axial (m) mode numbers for the interior double cylinder cavity

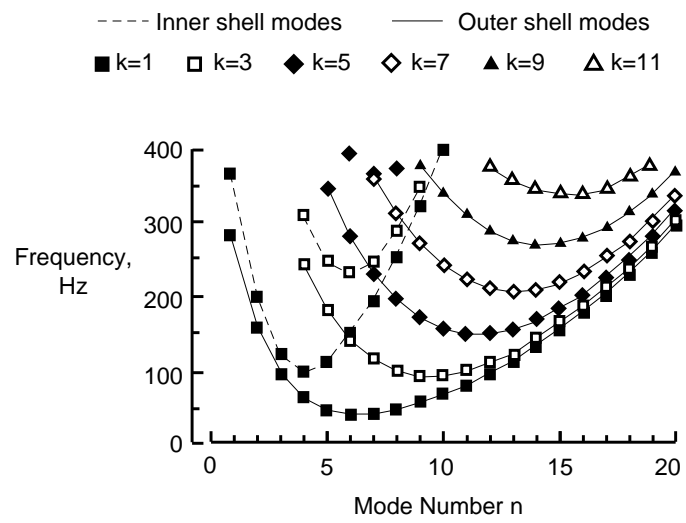

Figure 3. Structural circumferential (n) and axial (k) mode numbers for the unstiffened double wall cylinder configuration 


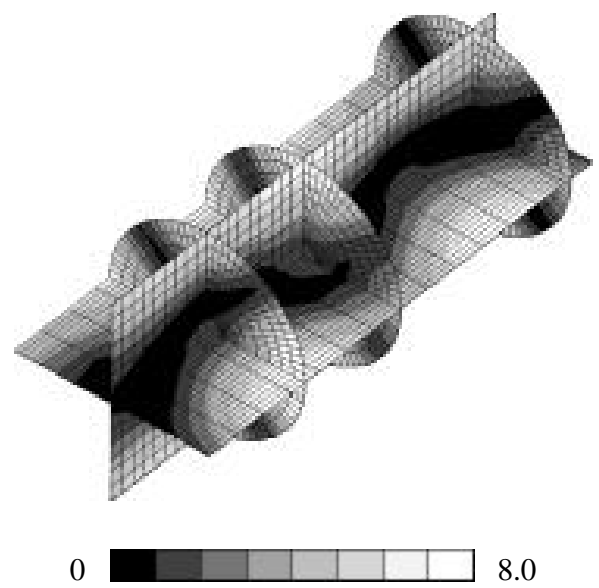

Figure 4. Normalized interior pressure distribution on the field recovery mesh for acoustic source monopole excitation at $213.8 \mathrm{~Hz}$

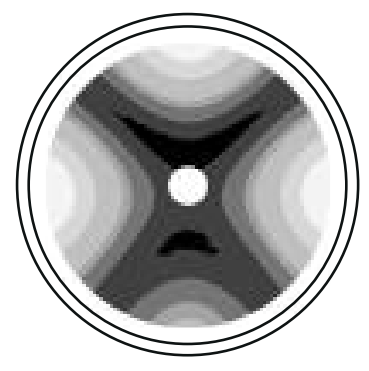

ख

0

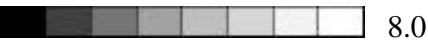

a) Center cross-sectional mesh

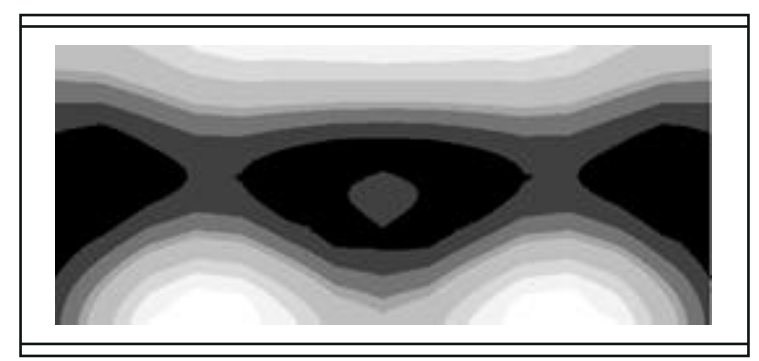

(3)

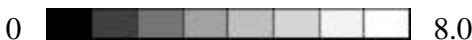

b) Horizontal mesh

Figure 5. Normalized pressure distribution inside the double cylinder excited by the acoustic source monopole at $213.8 \mathrm{~Hz}$

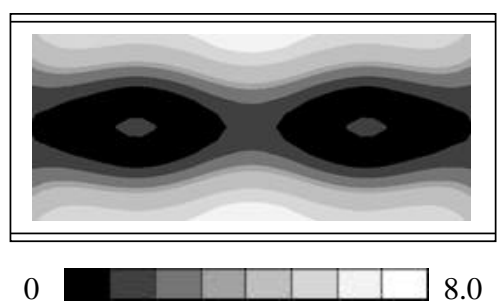

Figure 6. Normalized vertical pressure distribution inside the double cylinder excited by an acoustic source monopole at $213.8 \mathrm{~Hz}$

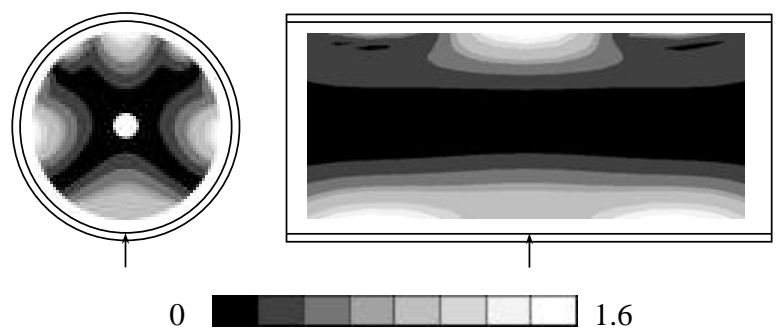

Figure 7. Normalized cross-sectional and horizontal interior pressure distribution due to control point force excitation (Configuration F1)

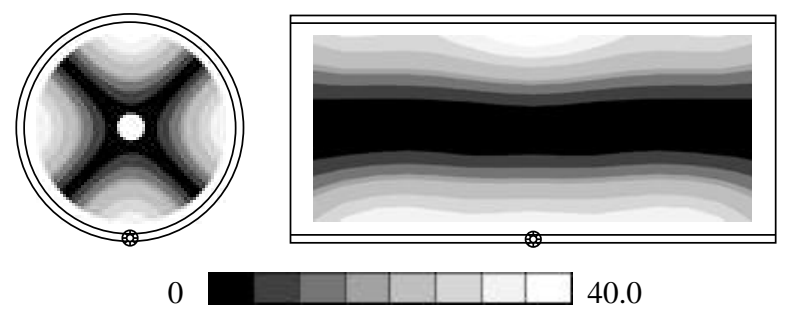

Figure 8. Normalized cross-sectional and horizontal interior pressure distributions due to acoustic control monopole excitation (Configuration M1)

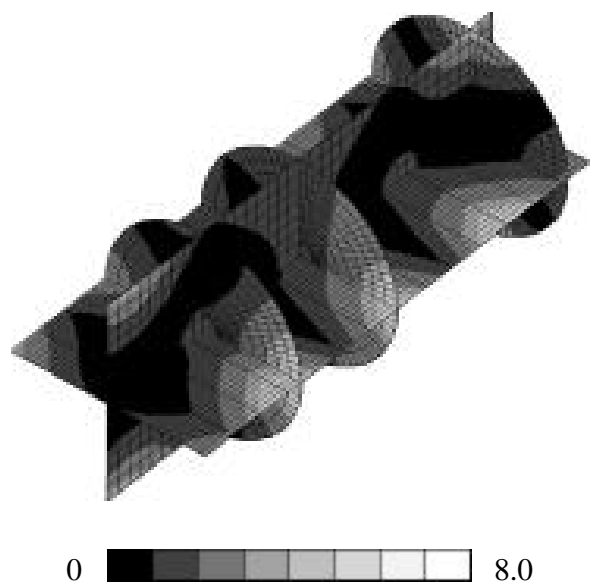

Figure 9. Normalized interior pressure distribution actively controlled by six point forces 


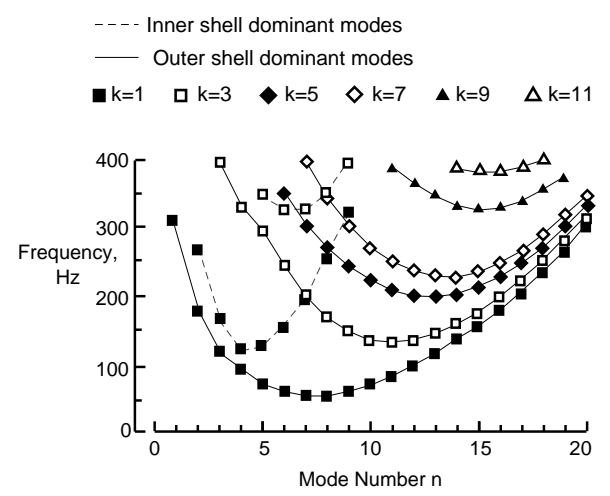

a) Stiffeners at $\mathrm{L} / 6$ from the end caps (RX)

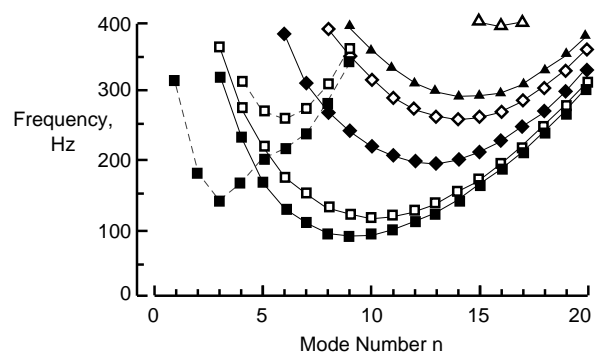

b) Stiffeners at L/3 from the end caps (XS)

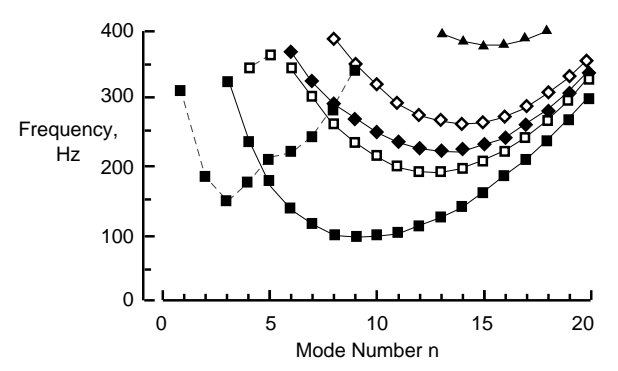

c) Stiffeners at $\mathrm{L} / 6$ and $\mathrm{L} / 3$ from the end caps (RS)

Figure 10. Structural circumferential (n) and axial (k) mode numbers for three stiffened configurations
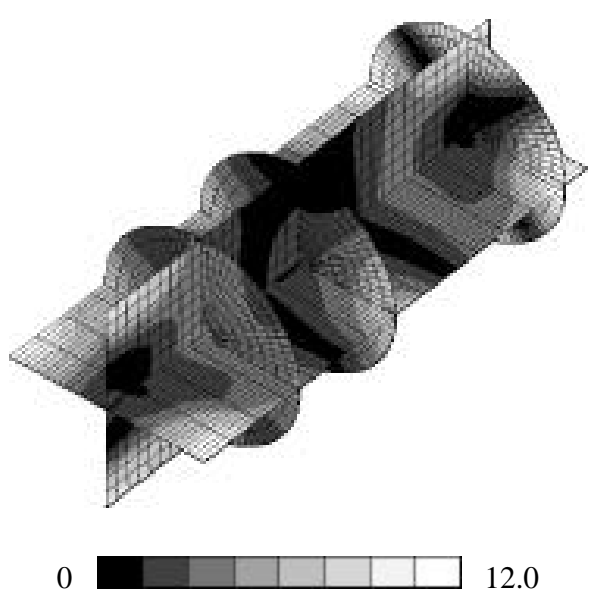

12.0

Figure 11. Normalized interior pressure distribution for acoustic source monopole excitation (RX)
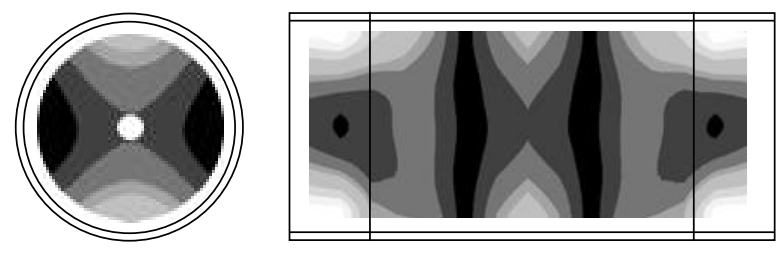

a) Acoustic source monopole excitation $\left(\mathrm{p}_{\max }=12.0\right)$
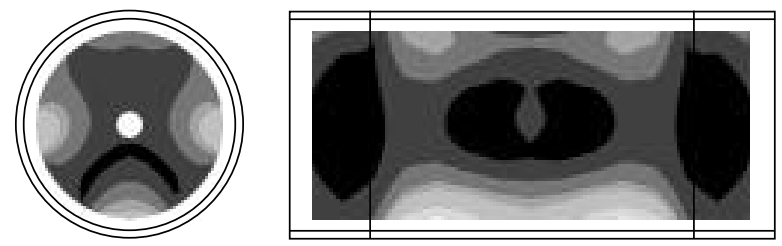

b) Control point force excitation $\left(\mathrm{p}_{\max }=1.6\right)$
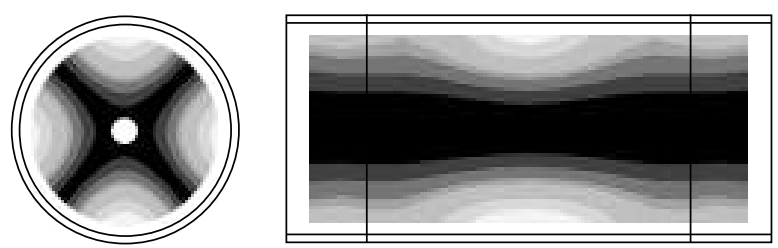

c) Acoustic control monopole excitation $\left(\mathrm{p}_{\max }=16.0\right)$

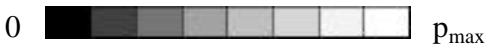

Figure 12. Normalized cross-sectional and horizontal interior pressure distributions (RX)
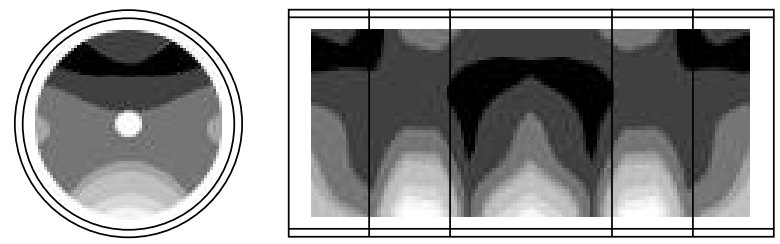

a) Acoustic source monopole excitation $\left(\mathrm{p}_{\max }=8.0\right)$
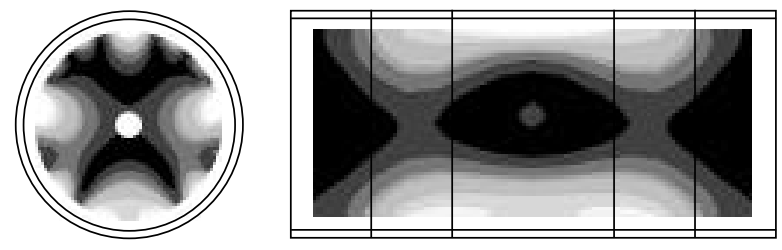

b) Control point force excitation $\left(\mathrm{p}_{\max }=1.6\right)$
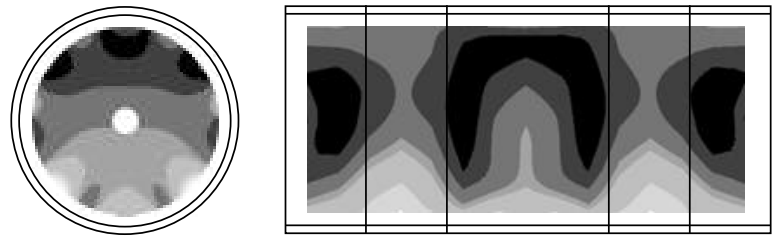

c) Acoustic control monopole excitation $\left(\mathrm{p}_{\max }=3.2\right)$

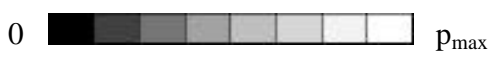

Figure 13. Normalized cross-sectional and horizontal interior pressure distributions (RS) 